\title{
Impact of feature extraction techniques on classification accuracy for EMG based ankle joint movements
}

\begin{abstract}
EMG based control becomes the core of the pros-theses, orthoses and rehabilitation devices in the recent research. Though the difficulties of using EMG as a control signal due to the complexity nature of this signal, the researchers employed the pattern recognition technique to overcome this problem. The EMG pattern recognition mainly consists of four stages; signal detection and preprocessing feature extraction, dimensionality reduction and classification. However, the success of any pattern recognition technique depends on the feature extraction and dimensionality reduction stages. In this paper time domain (TD) with 6th order auto regressive (AR) coefficients features and three techniques of dimensionality reduction; principal component analysis (PCA), uncorrelated linear discriminant analysis (ULDA) and fuzzy neighborhood preserving analysis with QR decomposition (FNPA-QR) were demonstrated. The EMG data were recorded from the below knee muscles of ten intactsubjects. Four ankle joint movements are classified using three classifiers; LDA, k-NN and MLP. The results show the superiority of TD\&6th AR with FNPA-QR and k-NN combination with $(96.20 \% \pm 4.1)$ accuracy.
\end{abstract}

Keyword: EMG; Feature extraction; Ankle joint movements; Classification accuracy 